\begin{tabular}{|c|c|}
\hline Title & $\begin{array}{l}\text { Characterization of GaA s Based Three Branch Nanowire Junction Devices by Light-Induced Local Conductance } \\
\text { Modulation Method }\end{array}$ \\
\hline Author(s) & Sato, Masaki; Kasai, Seiya \\
\hline Citation & $\begin{array}{l}\text { Japanese Journal of A pplied Physics(JJA P), 52(6S), 06GE08 } \\
\text { https://doi.org/10.7567/JA P.52.06GE08 }\end{array}$ \\
\hline Issue Date & $2013-06$ \\
\hline Doc URL & http:/hdl.handle.net/2115/66634 \\
\hline Rights & (c) 2013 The Japan Society of A pplied Physics \\
\hline Type & article (author version) \\
\hline File Information & JJAP2013_52_MN12077.pdf \\
\hline
\end{tabular}

Instructions for use 


\section{Characterization of GaAs-Based Three-Branch Nanowire Junction Devices by Light-Induced Local Conductance Modulation Method}

Masaki Sato and Seiya Kasai*

Graduate School of Information Science and Technology and Research Center for Integrated Quantum Electronics, Hokkaido University, Sapporo 060-0814, Japan

Nonlinear voltage transfer characteristics in GaAs-based three-branch nanowire junction (TBJ) devices were investigated by a light-induced local conductance modulation method. In this measurement system, the conductance in the device was locally increased by focused laser light irradiation. The nonlinear transfer curve was greatly changed when the laser light was irradiated on the positively biased branch. The conductance domain was found to exist at the end of the positively biased branch of the TBJ by scanning the light position. When a $\mathrm{SiN}_{x}$ thin layer was deposited on the nanowire surface, the surface potential was increased and the nonlinearity in the transfer curve was reinforced simultaneously. The obtained results suggest that the asymmetric channel depletion model is appropriate for the observed nonlinearity mechanism in the GaAs TBJ at room temperature.

*E-mail: kasai@rciqe.hokudai.ac.jp 


\section{Introduction}

The recent development of nanotechnology allows us to create various functional nanodevices. A semiconductor three-branch nano junction (TBJ) device is a typical and important example of such devices. The TBJ exhibits a unique nonlinear voltage transfer characteristic even with a simple structure ${ }^{1)}$. Various analog and digital circuits integrating TBJs have been demonstrated thus far, including NOR logic gates ${ }^{2}$, NAND logic gates $^{3-6)}$, frequency mixers ${ }^{7,8)}$, half adders ${ }^{9)}$, rectifiers ${ }^{10)}$, and flip-flops ${ }^{11,}$

12). The TBJ also has the capability of ultrahigh-speed operation up to THz frequency ${ }^{13)}$. For the design and control of the device and circuits, understanding of the mechanism for the nonlinear characteristic is an important issue. A pioneering work by $\mathrm{Xu}$ indicates that the nonlinear characteristic appears when the TBJ is operated in the ballistic transport regime ${ }^{1)}$. However, experimentally, it is also clearly observed at room temperature $(\mathrm{RT})^{14-18)}$ where the carrier transport should be in the nonballistic transport regime. The mechanism in such a case has not been clarified yet, although several hypotheses have been introduced, including those regarding the effective mean free path extension $^{16)}$ and the asymmetric channel depletion due to the surface potential ${ }^{19-26)}$.

In this study, to identify the model for the nonlinear mechanism in the TBJ at room temperature, we characterized the GaAs-based TBJ device by local conductance modulation by focused laser light irradiation. This method revealed the conductance domain in the device, which is an important factor for understanding the nonlinear characteristic. We also fabricated and characterized a $\mathrm{SiN}_{\mathrm{x}}$-deposited TBJ to clarify the effect of the surface potential on the nonlinear characteristic in terms of channel depletion. 


\section{Device Operation and Possible Mechanisms}

The TBJ with a typical measurement circuit is shown in Fig. 1(a). We consider the device with an n-type semiconductor. An equivalent circuit deduced from the structure is shown in Fig. 1(b). When voltage signals are applied to the left and right branches in push-pull fashion $\left(V_{\mathrm{INL}}=-V_{\mathrm{INR}}\right)$, the output voltage $V_{\mathrm{OUT}}$ in the center branch shows a bell-shaped nonlinear voltage transfer curve, as shown in Fig. 1(c). To explain such a nonlinear behavior in terms of the equivalent circuit in Fig. 1(b), we consider the formula of $V_{\text {OUT }}$ as

$$
V_{\mathrm{OUT}}=\frac{G_{+}-G_{-}}{G_{+}+G_{-}} V_{\mathrm{IN}},
$$

where $G_{+}$and $G_{-}$are the input branch conductance values with positive and negative biases, respectively. From the device configuration, the conductance in the input branches is expected to be the same and $V_{\text {OUT }}$ is expected to be zero. In turn, to explain the nonlinear curve, we must accept that $G_{+}$is always smaller than $G_{-}$.

The nonlinearity in the TBJ is understood by the asymmetric conductance that depends on the polarity of the input voltage. The original model explains it by the ballistic transport of electrons ${ }^{1)}$. In this model, the electrons emitted from the negatively biased branch reach another branch without scattering, as shown in Fig. 2(a). As a result, the resistance of the negatively biased branch is zero. Then, the output voltage follows that of the negatively biased branch, namely, $V_{\mathrm{OUT}}=-\left|V_{\mathrm{IN}}\right|$. This model is applicable to the low-temperature operation where the mean free path is longer than the device size. However, the nonlinear characteristic is observed at RT, which cannot be explained using this model. At present, there are two models for such a case. The mean free path extension model indicates that the effective mean free path is extended at a high electric field because the carrier velocity increases while the carrier relaxation time remains 
constant $^{16)}$. On the other hand, the asymmetric channel depletion model indicates that the potential difference between the surface and the biased branch causes asymmetric depletion in the channel, which is similar to the channel pinch off in a field-effect transistor (FET) with large drain bias. In the case of the n-type semiconductor, the channel depletion occurs in the positively biased branch, as shown in Fig. 2(b) ${ }^{19-26)}$. This means that $G_{+}$is small. The III-V semiconductor nanowire surface is known to behave similarly to a metal gate because high-density surface states fix the surface Fermi level at a certain energy level. From the size dependence of the nonlinear curve ${ }^{26}$ ) and its asymmetric change in the gate-controlled TBJ devices ${ }^{17,25)}$, we suppose that this model is applicable to the nonlinear characteristic at RT. To verify the applicability of this model, the identification of the existence of the conductance domain and its portion is necessary. The effect of the surface should also be clarified.

\section{Experimental Procedure}

In this study, GaAs-based TBJ devices were fabricated and characterized. The nanowire channel was formed on an $\mathrm{AlGaAs} / \mathrm{GaAs}$ heterostructure on a (001) semi-insulating GaAs substrate by EB lithography and wet chemical etching. A $\mathrm{Ni} / \mathrm{Ge} / \mathrm{Au} / \mathrm{Ni} / \mathrm{Au}$ ohmic contact was formed on each branch. Figure 3(a) shows a scanning electron microscopy (SEM) image of a fabricated device. The two-dimensional electron gas (2DEG) density was $7.8 \times 10^{11} \mathrm{~cm}^{2}$. The 2 DEG mobility $\left(\mu_{\mathrm{e}}\right)$ values were $7,100 \mathrm{~cm}^{2} \mathrm{~V}^{-1} \mathrm{~s}^{-1}$ at $300 \mathrm{~K}$ and $11,000 \mathrm{~cm}^{2} \mathrm{~V}^{-1} \mathrm{~s}^{-1}$ at $77 \mathrm{~K}$. The corresponding mean free paths were $100 \mathrm{~nm}$ at $300 \mathrm{~K}$ and $1600 \mathrm{~nm}$ at $77 \mathrm{~K}$. To identify the conductance domain position distinctly, we designed a relatively large device. The input nanowire length was $20 \mu \mathrm{m}$ and the output nanowire length was $2.5 \mu \mathrm{m}$. The 
nanowire directions were $<-110>$ for input branches and $<110>$ for output branches. In the case of the GaAs channel, the electron transport properties including the mobility, mean free path, and density of states do not depend on the nanowire direction, since the Fermi surface of GaAs has a spherical symmetry and the effective mass of the electron is isotropic. The width and cross section of the nanowire mainly affect the conductivity. The nanowire widths were $300 \mathrm{~nm}$ for the input branch and $500 \mathrm{~nm}$ for the output branch. The nanowire was larger than the mean free path and the device operated in the nonballistic transport regime at RT. To clarify the effect of the surface potential, we also prepared devices with and without a $\mathrm{SiN}_{\mathrm{x}}$ layer on their surface. The $\mathrm{SiN}_{\mathrm{x}}$ layer is known to change the surface potential in GaAs-based materials ${ }^{27,28)}$. The $\mathrm{SiN}_{\mathrm{X}}$ layer was deposited by electron cyclotron resonance chemical vapor deposition (ECR-CVD) at a substrate temperature of $260{ }^{\circ} \mathrm{C}$. The gas flow conditions were a $\mathrm{SiH}_{4}$ gas flow rate of $30 \mathrm{sccm}$ and a $\mathrm{N}_{2}$ gas flow rate of $1 \mathrm{sccm}$. The measured film thickness was $30 \mathrm{~nm}$.

To find the conductance domain and its effect on the nonlinear characteristic, we developed the light-induced local conductance modulation measurement system shown in Fig. 3(b). A focused laser light was irradiated on the device. The diameter of the laser spot was $5 \mu \mathrm{m}$ and the wavelength was $658 \mathrm{~nm}$. The laser light excited carriers and modulated the conductance locally. The sample position could be controlled with a $10 \mathrm{~nm}$ resolution in this system. All measurements were performed at RT.

\section{Results and Discussion}

\subsection{Effect of focused light irradiation on branch conductance}

To confirm the local conductance modulation in the developed system, we measured the $I-V$ characteristics of the device in the beginning. Figure 4(a) shows the 
measured $I-V$ characteristics under dark and light irradiation conditions. The laser light was irradiated at the end of the left branch. The significant current increase was observed only when the laser light was irradiated on the positively biased branch. The results confirmed the local conductance modulation in the present system and also indicated the existence of the conductance domain in this portion. A small increase in current was also observed even in the branch without light irradiation, as shown in Fig. 4(b). This was because the photogenerated carriers in the laser-irradiated branch diffused to the current channel portion. The increase in conductance was $22 \mu \mathrm{S}$. The estimated increase in carrier density, $\Delta n$, was $1.9 \times 10^{10} \mathrm{~cm}^{-2}$. Theoretically, in the case of $658 \mathrm{~nm}$ light, the number of generated photons was $8.6 \times 10^{16} \mathrm{~cm}^{-2} \mathrm{~s}^{-1}$ considering the reflectance of 0.34 for GaAs and the laser power of $0.102 \mathrm{~mW}$. The increase in carrier density is estimated using

$$
\Delta n=\eta \tau f,
$$

where $\tau$ is the carrier lifetime, $\eta$ is the quantum efficiency, and $f$ is the photon flux density. $\tau$ was estimated to be 105 ns by taking into account the surface recombination on the bulk structure ${ }^{29)}$. Assuming $\eta=30 \%$, we obtained $\Delta n=1.3 \times 10^{12} \mathrm{~cm}^{-2}$ and the increase in conductance was estimated to be $1.5 \mathrm{mS}$. This value is two orders of magnitude larger than the experimental one. The difference is attributed to the small effective quantum efficiency due to the large surface recombination of the GaAs nanowire ${ }^{30)}$.

\subsection{Nonlinear voltage transfer characteristics}

Figure 5 shows the measured $V_{\mathrm{OUT}^{-}} V_{\mathrm{INL}}$ characteristics with and without laser light irradiation. The output voltage increased when the laser light was irradiated on the 
positively biased branch. In contrast, the output decreased when the laser light was irradiated on the negatively biased branch. The observed behaviors were explained by the increase in conductance in the laser-irradiated branch in terms of Eq. (1). The polarity of the output voltage was changed from negative to positive by the light on the positively biased branch. This was because the magnitude relationship between the conductance in the left and right branches was switched and the output branch potential followed the positively biased branch. The increase in conductance from the result was estimated to be $11 \mu \mathrm{S}$, which was in agreement with that from the $I-V$ characteristics. This result indicates that the asymmetry of the input nanowire conductance caused the nonlinearity in the voltage transfer curve.

\subsection{Conductance domain}

To determine the position of the conductance domain and its relationship with the nonlinear curve, the laser light position dependences of the output voltage and the input-branch current $I_{\mathrm{IN}}$ were characterized. The measurement system is schematically shown in Fig. 6(a). In these measurements, constant positive and negative voltages were applied to the left and right branches, respectively $\left(V_{\mathrm{INL}}=+1 \mathrm{~V}, V_{\mathrm{INR}}=-1 \mathrm{~V}\right)$. Figures 6(b) and 6(c) show the laser position dependences of $V_{\text {OUT }}$ and $I_{\mathrm{IN}}$, respectively. The output voltage increased as the laser position was moved from right $(x=10 \mu \mathrm{m})$ to left $(x=-10 \mu \mathrm{m})$. The current also increased similarly to the output voltage. The highest voltage and current were observed at the end of the positively biased nanowire at $x=$ $-10 \mu \mathrm{m}$. These results indicate that the lowest conductance portion was the end of the positively biased nanowire. $V_{\text {OUT }}$ showed a minimum value at $x=2.5 \mu \mathrm{m}$. This was because the conductance in the negatively biased branch, $G_{-}$, increased and it enhanced 
the nonlinearity, as indicated by Eq. (1). $V_{\text {OUT }}$ increased when the laser position was greater than $2.5 \mu \mathrm{m}$, since the light spot gradually left the nanowire and transferred to the wide channel portion, and the situation approached the dark condition.

We estimated the change in conductance in the two input branches as a function of the laser-irradiated position. Input branch conductance is expressed by

$$
G_{+}=\frac{I_{\mathrm{IN}}}{V_{\mathrm{INL}}-V_{\mathrm{OUT}}}, G_{-}=\frac{I_{\mathrm{IN}}}{V_{\mathrm{OUT}}-V_{\mathrm{INR}}} .
$$

The calculated result is shown in Fig. 7. Note that the largest conductance change occurs when the laser is irradiated on the conductance domain, since the lowest conductance dominates the current. Therefore, we could determine the position of the conductance domain from the plot in Fig. 7. The largest change in $G_{+}$was obtained when the laser was irradiated at the end of the positively biased nanowire, $x=-10 \mu \mathrm{m}$. Thus, the conductance domain existed at the end of the positively biased nanowire.

\subsection{Mechanism for conductance domain formation}

To clarify the mechanism of the formation of the conductance domain, we investigated the effect of the nanowire surface on the electric characteristics in the TBJ. Figure 8(a) shows the currents in the nanowire before and after the $\operatorname{SiN}_{x}$ deposition. The current decreased after the $\mathrm{SiN}_{\mathrm{x}}$ deposition. This showed the increase in surface potential that promoted the channel depletion. Figure 8(b) shows the voltage transfer curves before and after the $\mathrm{SiN}_{x}$ deposition. The reinforcement of the nonlinearity was observed in the device after the $\operatorname{SiN}_{x}$ deposition. Therefore, the surface condition affected the nonlinearity in the TBJ. The study on the electric field concentration in MESFETs $^{31)}$ showed that the large surface potential of the channel semiconductor shifted the position of the high field to the drain electrode. In the case of the TBJ, the 
increased surface potential enhanced the channel depletion in the positively biased branch similar to the drain in a FET and the conduction was decreased and the nonlinearity was reinforced. Considering the position of the conductance domain, the results suggest that the asymmetry channel depletion model is feasible for the nonlinear characteristic of the TBJ in the nonballistic transport regime.

\section{Conclusion}

Nonlinear voltage transfer characteristics in GaAs-based three-branch nanowire junction (TBJ) devices were investigated by a light-induced local conductance modulation method. The nonlinear transfer curve greatly changed only when the laser was irradiated on the positively biased branch. The conductance domain was found to

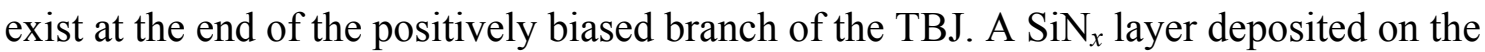
TBJ increased the surface potential and reinforced the nonlinearity in the transfer curve. The obtained results indicate that the asymmetric channel depletion model is appropriate for the observed nonlinearity mechanism in the GaAs TBJ at room temperature.

\section{Acknowledgements}

We thank Dr. T. Maemoto of Osaka Institute of Technology for various useful discussions. We also thank Professor T. Fukui and Professor T. Hashizume of Hokkaido University for their continuous support. This work was partly supported by a Grant-in-Aid for Scientific Research (No. 22310084), the Japan Society for the Promotion of Science. 


\section{References}

1) H. Q. Xu: Appl. Phys. Lett. 78 (2001) 2064.

2) C. R. Müller, L. Worschech, P. Höpfner, S. Höfling, and A. Forchel: IEEE Electron Device Lett. 28 (2007) 859.

3) H. Q. Xu, I. Shorubalko, D. Wallin, I. Maximov, P. Omling, L. Samuelson, and W. Seifert: IEEE Electron Device Lett. 25 (2004) 164.

4) H. Q. Xu: Nat. Mater. 4 (2005) 649.

5) S. Reitzenstein, L. Worschech, C. R. Müller, and A. Forchel: IEEE Electron Device Lett. 26 (2005) 142.

6) S. F. Abd Rahman, D. Nakata, Y. Shiratori, and S. Kasai: Jpn. J. Appl. Phys. 48 (2009) 06FD01.

7) J. Sun, D. Wallin, P. Brushiem, I. Maximov, Z. G. Wang, and H. Q. Xu: Nanotechnology 18 (2007) 195205.

8) I. Shorubalko, H. Q. Xu, I. Maximov, D. Nilsson, P. Omling, L. Samuelson, and W. Seifert: IEEE Electron Device Lett. 23 (2002) 377.

9) S. Reitzenstein, L. Worschech, and A. Forchel: IEEE Electron Device Lett. 25 (2004) 462.

10) L. Bednarz, Rashmi, B. Hackens, G. Farhi, V. Bayot, and I. Huynen: IEEE Trans. Electron Devices 4 (2005) 576.

11) J. Sun, D. Wallin, I. Maximov, and H. Q. Xu: IEEE Electron Device Lett. 29 (2008) 540.

12) H. Shibata, Y. Shiratori, and S. Kasai: Jpn. J. Appl. Phys. 50 (2011) 06 GF03.

13) S. Bollaert, A. Cappy, Y. Roelens, J. S. Gallo, C. Gardes, Z. Teukam, X. Wallart, J. Mateos, T. González, B. G. Vasallo, B. Hackens, L. Berdnarz, and I. Huynen: Thin 
Solid Films 515 (2007) 4321.

14) I. Shorubalko, H. Q. Xu, I. Maximov, P. Omling, L. Samuelson, and W. Seifert: Appl. Phys. Lett. 79 (2001) 1384.

15) I. Maximov, P. Carlberg, I. Shorubalko, D. Wallin, E-L. Sarwe, M. Beck, M. Graczyk, W. Seifert, H.Q. Xu, L. Montelius, and L. Samuelson: Microelectron. Eng. 67 (2003) 196.

16) D. Wallin, I. Shorubalko, H. Q. Xu, and A. Cappy: Appl. Phys. Lett. 89 (2006) 092124.

17) T. Nakamura, S. Kasai, Y. Shiratori, and T. Hashizume: Appl. Phys. Lett. 90 (2007) 102104.

18) M. Frimmer, J. Sun, I. Maximov, and H. Q. Xu: Appl. Phys. Lett. 93 (2008) 133110.

19) J. Mateos, B. G. Vsasallo, D. Pardo, T. González, J.-S. Galloo, S. Bollaert, Y. Roelens, and A. Cappy: Nanotechnology 14 (2003) 117.

20) J. Mateos, B. G. Vsasallo, D. Pardo, T. González, E. Pichonat, J.-S. Galloo, S. Bollaert, Y. Roelens, and A. Cappy: IEEE Electron Device Lett. 25 (2004) 235.

21) J. Mateos, B. G. Vsasallo, D. Pardo, T. González, J.-S. Galloo, S. Bollaert, Y. Roelens, and A. Cappy: IEEE Trans. Electron Devices 50 (2003) 1897.

22) I. Iñiguez-de-la-Torre, J. Mateos, T. González, D. Pardo, J.-S. Galloo, S. Bollaert, Y. Roelens, and A. Cappy: Semicond. Sci. Technol. 22 (2007) 663.

23) T. Sadi, F. Dessenne, and J. Thobel: J. Appl. Phys. 105 (2009) 053707.

24) I. Iñiguez-de-la-Torre, J. Mateos, Y. Roelens, C. Gardés, S. Bollaert, and T. González: Nanotechnology 22 (2011) 445203.

25) S. Kasai, T. Nakamura, S. F. Abd Rahman, and Y. Shiratori: Jpn. J. Appl. Phys. 49 
(2008) 4958.

26) D. Nakata, H. Shibata, Y. Shiratori1, and S. Kasai: Jpn. J. Appl. Phys. 49 (2010) 06GG03.

27) E. Y. Chang, G. T. Cibuzar, and K. P. Pande: IEEE Trans. Electron Devices 35 (1988) 1412.

28) S. Pal and D. N. Bose: Appl. Surf. Sci. 181 (2001) 179.

29) J. M. Borrego and S. K. Ghandhi: Solid-State Electron. 33 (1990) 733.

30) C. Chang, C. Chi, M. Yao, N. Huang, C. Chen, J. Theiss, A. W. Bushmaker, S. LaLumondiere, T. Yeh, M. L. Povinelli, C. Zhou, P. D. Dapkus, and S. B. Cronin: Nano Lett. 12 (2012) 4484.

31) H. Mizuta, K. Yamaguchi, and S. Takahashi: IEEE Trans. Electron Devices 34 (1987) 2027. 


\section{Figure captions}

Fig. 1. Three-branch nanowire junction (TBJ) device: (a) measurement circuit, (b) equivalent circuit of the device, and (c) typical voltage transfer characteristic.

Fig. 2. (a) Ballistic transport model and (b) asymmetric channel depletion model.

Fig. 3. (a) SEM image of a fabricated GaAs TBJ device and (b) a light-induced local conductance modulation measurement system.

Fig. 4. $I-V$ characteristics with laser light irradiation: (a) irradiation on the current path side and (b) irradiation on the outside of the path.

Fig. 5. $V_{\mathrm{OUT}}-V_{\mathrm{INL}}$ characteristics with and without the laser light irradiation.

Fig. 6. (a) Measurement system for conductance domain identification and (b) laser light positional dependences of (b) $V_{\text {OUT }}$ and (c) $I_{\mathrm{IN}}$.

Fig. 7. Evaluated conductance values in the left and right branches.

Fig. 8. (a) $I-V$ and (b) $V_{\text {OUT }}-V_{\text {INL }}$ characteristics before and after $\operatorname{SiN}_{\mathrm{x}}$ deposition. 


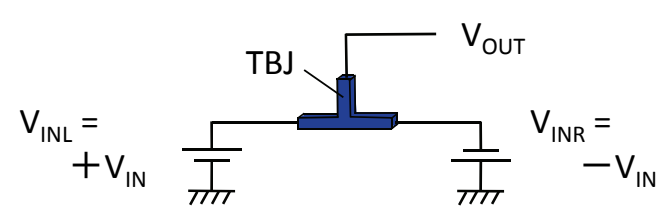

(a)

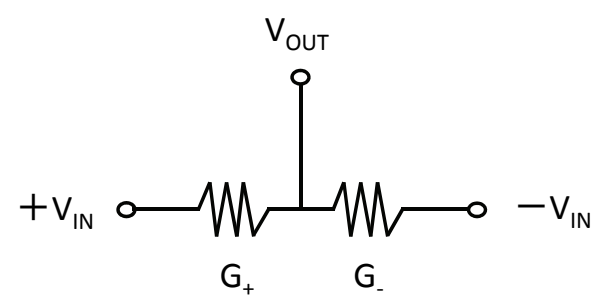

(b)

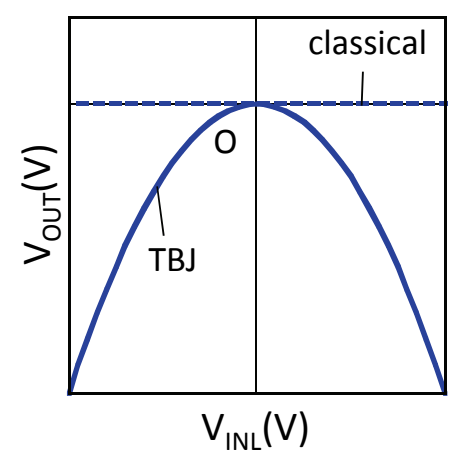

(c)

Sato et al., Figure. 1. 


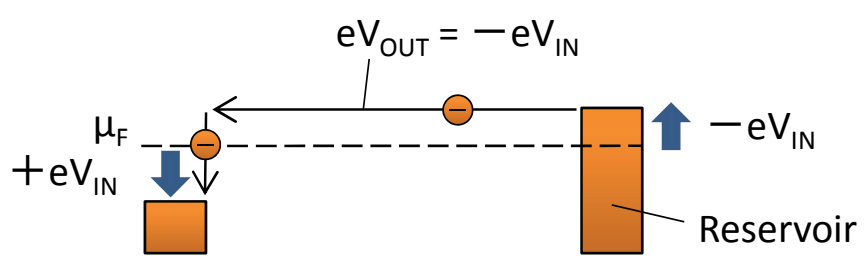

(a)

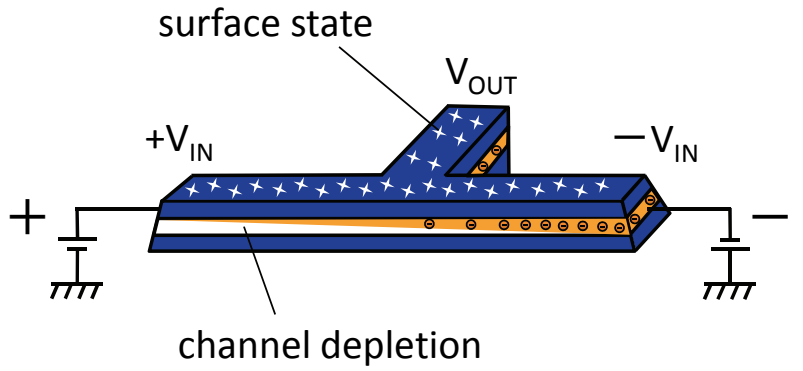

(b)

Sato et al., Figure. 2. 


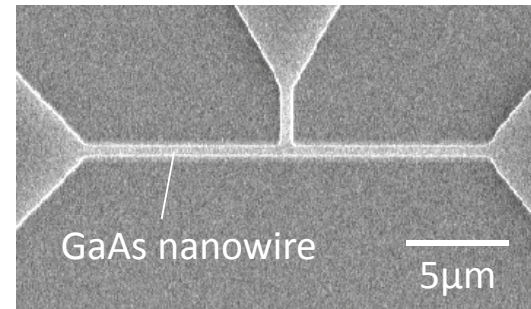

(a)

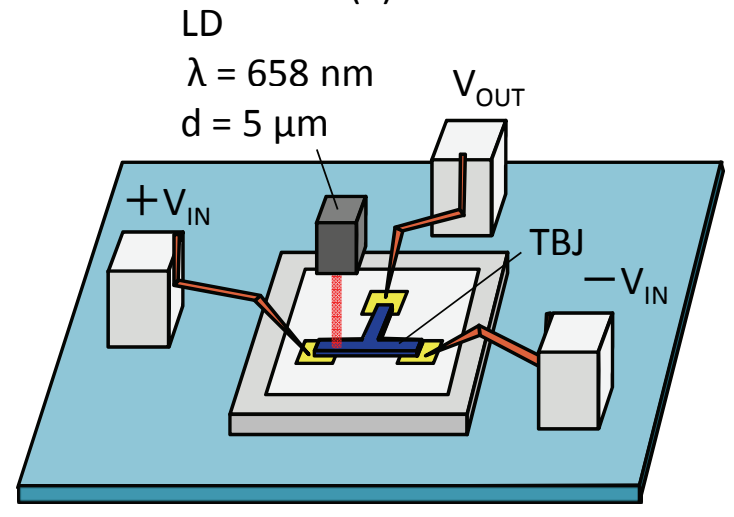

(b)

Sato et al., Figure. 3. 


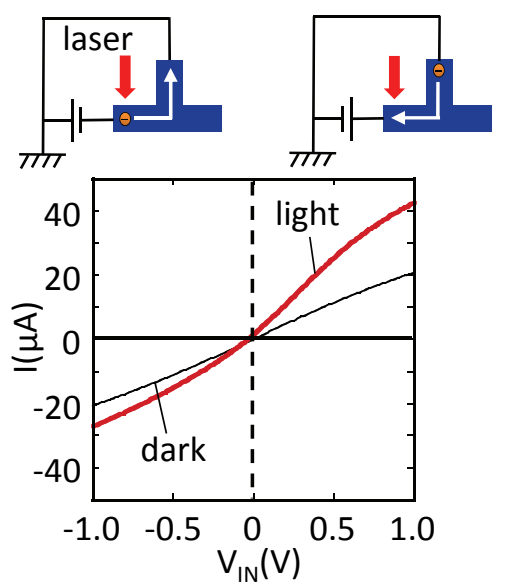

(a)
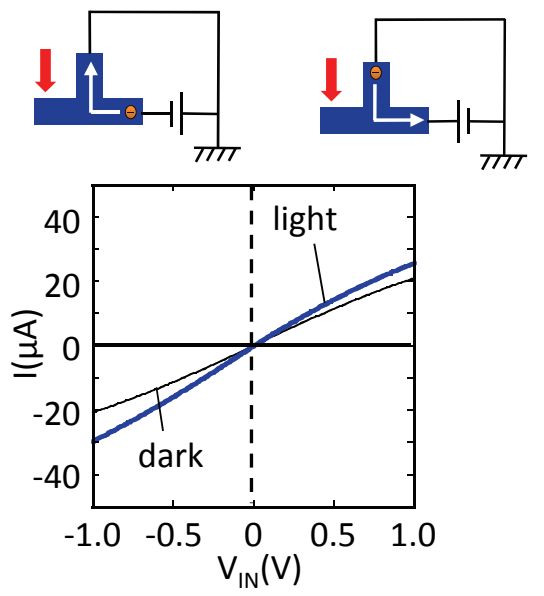

(b)

Sato et al., Figure. 4. 


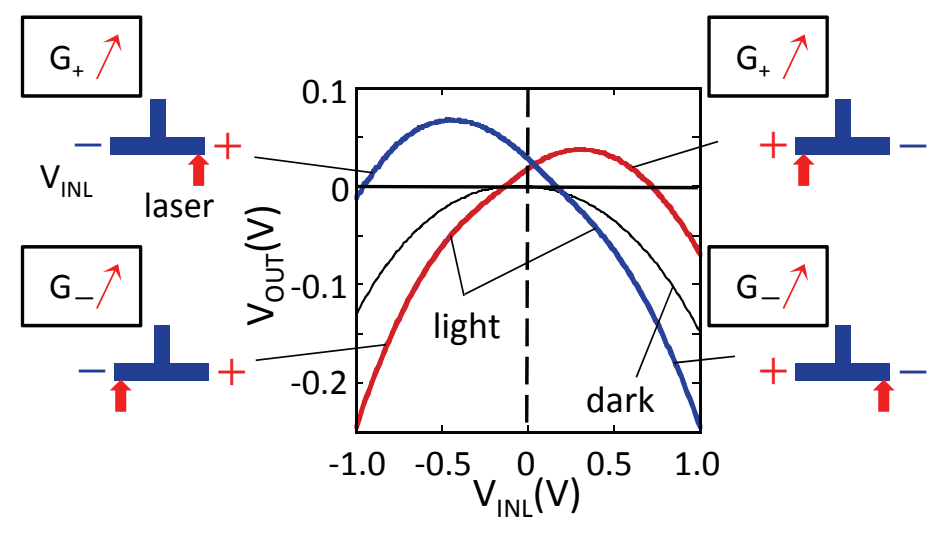

Sato et al., Figure. 5. 


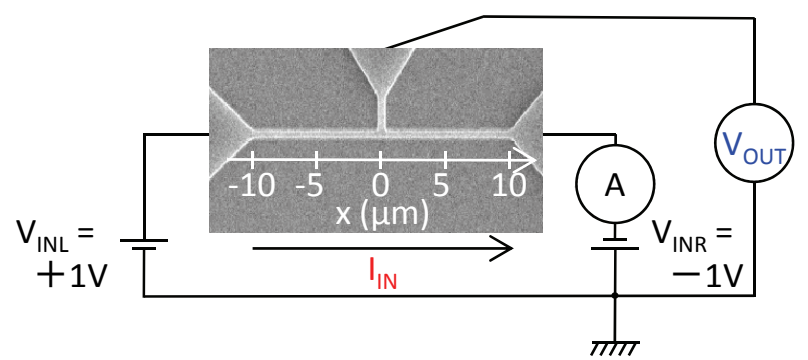

(a)

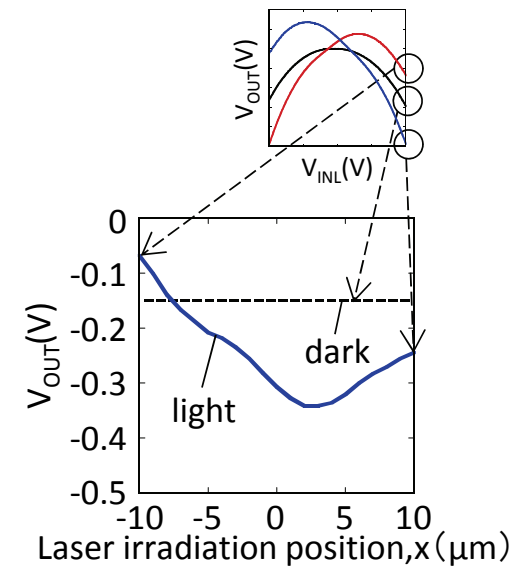

(b)

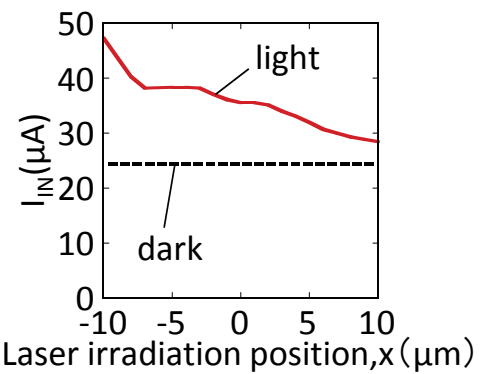

(c)

Sato et al., Figure. 6. 


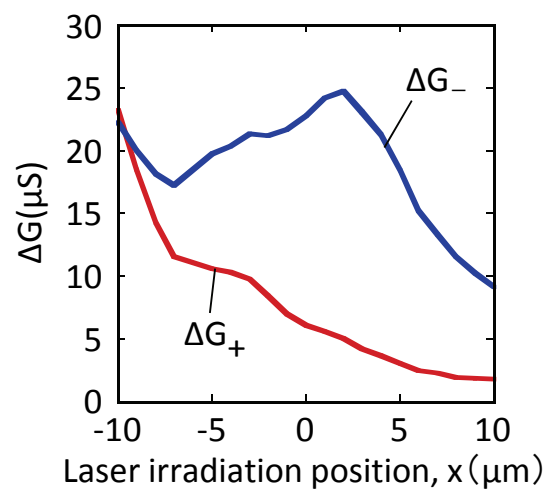

Sato et al., Figure. 7. 


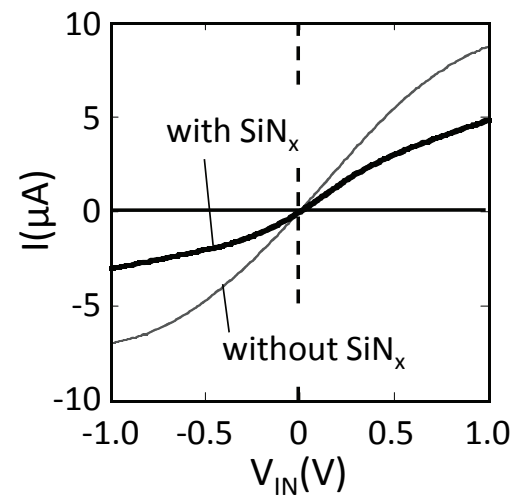

(a)

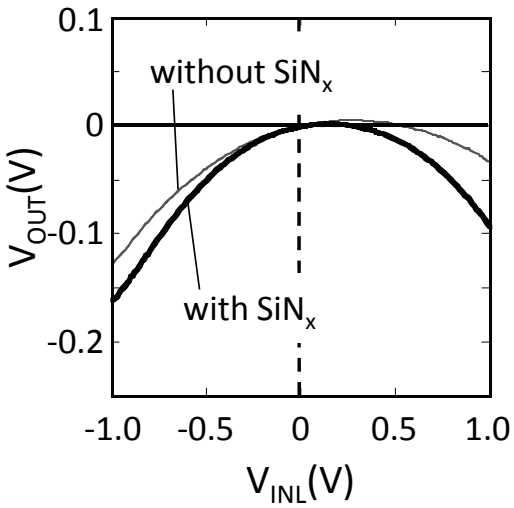

(b)

Sato et al., Figure. 8. 